\title{
Size scaling in two-dimensional pictorial arrays
}

\author{
JOHN UHLARIK, RICHARD PRINGLE, KEVIN JORDAN, and GIOVANNI MISCEO \\ Kansas State University, Manhattan, Kansas 66506
}

\begin{abstract}
The study examined effects of instructional sets tobjective, phenomenal, projective, and retinal) on the judged sizes of blocks placed at various "distances" in a pictorial array. Magnitude estimations of size were consistent with previous studies of size constancy in three-dimensional arrays. Chronometric analyses indicated that reaction time increased with distal size, but was not affected by perceived distance. The results suggested that size was scaled relative to a perceptual unit. Instructions affected the nature of the scale unit (proximal vs. distal), but not the scaling process itself.
\end{abstract}

In an attempt to examine the mental processes that underlie the psychophysical scaling of size, Hartley (1977) measured the response times associated with magnitude estimations of line lengths and found that reaction times increased linearly with the number of standard units contained in the comparison lines. He proposed that observers judged length by counting or "laying off" a mental image of the standard against the comparison. In the present study, this reaction time approach was extended to the scaling of sizes that vary in perceived distance. Specifically, a method of magnitude estimation was used to directly assess apparent size in a twodimensional pictorial array. The mental transformations that underlie size estimation and size constancy were then investigated by examining the relationship between response time and perceived size.

The extent to which perceived size remains constant with variations in distance is profoundly dependent on the judgmental attitude adopted by the observer. For example, if an observer is given phenomenal or "first look" instructions, variation in distance usually has no effect on judged size. However, if the observer is given objective or projective instructions, judged size will increase or decrease with distance, respectively (see Carlson, 1977, for review). Any attempt to model the mental processes that underlie size scaling and size constancy must ultimately incorporate these effects of judgmental set. Hence, the present experiment examined the response

This research was conducted while John Uhlarik was a Summer Research Fellow at Kansas State University and was supported by the Bureau of General Research, Kansas State University. Richard Pringle is now at Goucher College. A version of this article was presented at the Psychonomic Society meetings, San Antonjo, Texas, November 1978. Requests for reprints should be sent to John Uhlarik, Department of Psychology, Kansas State University, Manhattan, Kansas 66506. times associated with magnitude estimations of size for a variety of instructional conditions.

\section{METHOD}

Subjects

Forty-eight introductory psychology students served as subjects for course credit. Normal visual acuity or vision corrected to $20 / 30$ was a prerequisite for serving in the experiment.

\section{Stimuli}

It has been previously demonstrated that relatively accurate distance perception is maintained in both photographs and perspective drawings of three-dimensional scenes (e.g., Hagen. 1978; Hagen \& Jones, 1978; Smith \& Gruber, 1958; Smith, Smith, \& Hubbard, 1958). In an attempt to eliminate systematic reaction time effects due to accommodation and vergence eye movements necessary to fixate objects at different distances, all viewing conditions in the present study involved only pictorial cues for depth that could be displayed in a single depth plane on a twodimensional frontal surface. Each stimulus consisted of a twodimensional pictorial representation of a white block on a receding surface. Figure 1 shows an achromatic example of the photographic stimulus array. The complete set of stimuli consisted of color photographic slides that represented the factorial combination of six block widths and four distances along the receding surface. All six blocks had distal heights and depths of $3.8 \mathrm{~cm}$. However, the distal widths varied as indicated in Table 1, which shows the factorial combination of the six block widths and four physical distances. The distances were measured from the camera lens to the surface gradient. Because of foreshortening, the four distances were not evenly distributed along the vertical extent of the two-dimensional pictorial array. To achieve a more uniform distribution, filler stimuli, consisting of the six sizes at a distance of $2.25 \mathrm{~m}$, were also prepared.

The stimulus array was photographed with a $35-\mathrm{mm}$ single lens reflex camera with a $50-\mathrm{mm} / 1.4$ macro lens. The camera was mounted with the lens $.7 \mathrm{~m}$ above the textured array. Slides were made from Kodak high-speed Ektachrome (E6) film with a lens opening of $f / 22$, which allowed the stimulus blocks to be adequately focused at all distances.

The three-dimensional textured array consisted of two $.92 \times$ $7.3 \mathrm{~m}$ panels of textured cloth joined by a visible seam. Each panel had a slight crease down its middle. The cloth was dark blue with white polka dots, $6 \mathrm{~mm}$ in diameter, uniformly distributed with a density of $.6 / \mathrm{cm}^{2}$. The array was placed on the floor of an evenly illuminated room. Because the lighting came from directly above and extended uniformly throughout the array, 


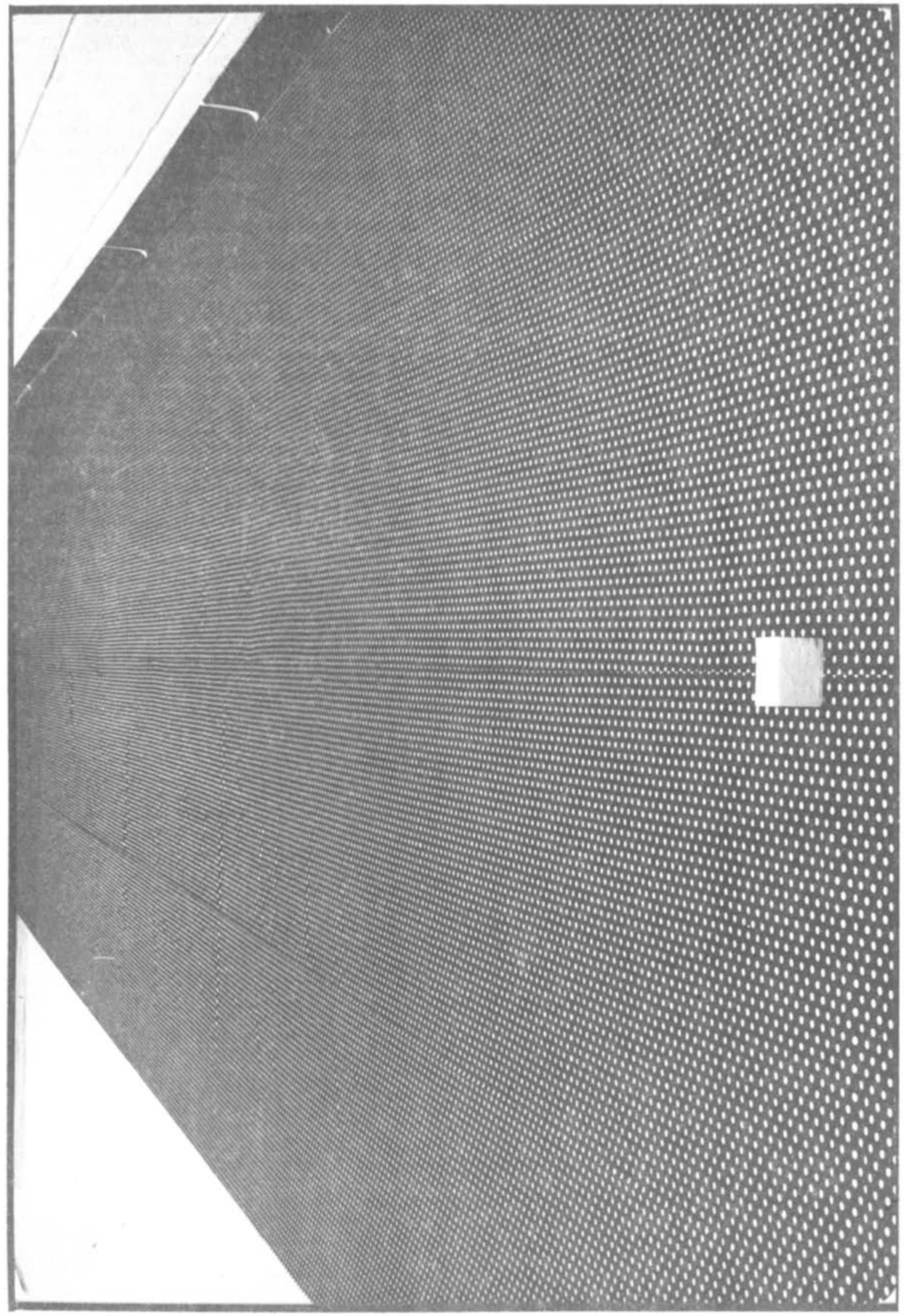


Table 1

Proximal Stimulus Sizes (in Degrees of Visual Angle) Produced by the Factorial Combination of Distal Stimulus Widths (in Centimeters) and Distances of Stimuli (in Meters) from Camera

\begin{tabular}{rrrrr}
\hline & \multicolumn{4}{c}{ Distance } \\
\cline { 2 - 5 } Width & 1.5 & 3.0 & 4.5 & 6.0 \\
\hline 5 & $1.9^{*}$ & 1.0 & .6 & .5 \\
10 & 3.8 & 1.9 & 1.3 & 1.0 \\
20 & 7.6 & 3.8 & 2.5 & 1.9 \\
30 & 11.3 & 5.7 & 3.8 & 2.9 \\
40 & 14.9 & 7.6 & 5.1 & 3.8 \\
50 & 18.4 & 9.5 & 6.3 & 4.8 \\
\hline
\end{tabular}

*The 5-cm-wide stimulus at a distance of $1.5 \mathrm{~m}$ was defined as the 10-unit standard.

no shadows were cast by the blocks. However, the lighting produced differential reflectance from the surface of the stimulus blocks, which provided additional depth cues.

\section{Procedure}

Stimuli were presented by a random-access slide projector in conjunction with a rear projection screen $2.2 \mathrm{~m}$ from the lens of the projector and $.9 \mathrm{~m}$ from the observer. The projected image was $.4 \mathrm{~m}$ high and $.5 \mathrm{~m}$ wide. The visual angles subtended by the blocks as projected on the screen duplicated those that would have been subtended if an observer were substituted for the camera in the three-dimensional photographic field. The projection screen was viewed binocularly through a viewing hood that restricted the field of vision to an elliptical area (41 deg wide and $30 \mathrm{deg}$ high) encompassing the entire pictorial array.

Each observer was tested individually. The observer initiated a trial by pressing a switch which both started a timer and opened a shutter on the slide projector. Verbal size estimates activated an electronic voice key that both stopped the timer and closed the projection shutter. Prior to the initiation of each trial, the observer fixated a luminous 6-mm point on the projection screen. To control for any possible systematic differences in visual search time involved in scanning the array, half of the observers in each instructional group fixated a dot at the middle of the top edge of the pictorial array and the other half fixated a dot at the middle of the bottom edge. Twenty-six ( 24 test and 2 filler) stimuli were presented in different random orders for six replications. The first replication was considered practice and was not used in the final data analysis.

\section{Instructions}

The method of magnitude estimation was used to obtain judgments of size. A direct scaling technique was used because observers can make the required judgments quickly and easily, and furthermore, it allows for the integration of issues related to size constancy into the broader framework of the perception of size (see Teghtsoonian \& Teghtsoonian, 1970, for a discussion of the use of magnitude estimation to study size constancy).

The experimenter assigned a width of "10 units" to the $5-\mathrm{cm}$ wide block at the closest distance $(1.5 \mathrm{~m})$, and the observer assigned proportional numbers to the widths of the various comparison blocks. The subjects were told that "this would mean that a comparison block that is twice as wide would be assigned a number twice as great; three times as wide, three times as great; half as wide, half as great, and so forth." Observers were encouraged to use fractions, decimals, or whole numbers as long as their judgments were proportional to the 10-unit standard, and to respond as rapidly and as accurately as possible.

All subjects receiving objective-size, phenomenal-size, or projective-size instructions were given the following general instructions:
In this experiment, I would like you to judge the size of various objects that will appear to be at various distances. These objects are blocks of different widths that will be presented on the screen that you see through the viewer. Although the screen will always be the same distance from you, you can see that the surface of the sample slide appears to recede from you in depth. You can think of this surface as a road or sidewalk going off into the distance, or as a carpet on a floor. Imagine it in the way that gives you the greatest feeling of depth, or three-dimensionality. I would like you to judge the width of the block that will appear on each slide. These blocks will appear to be at different distances on various trials.

Specific instructions were adapted from Carlson (1962) as follows:

Objective size. You should try to estimate what the actual physical width of each block would be along such a road or sidewalk. For example, call the width of the sample block in the screen " 10 units." If you see a block of the same width but at a different distance, it should also be judged to be 10 units wide. Here is how the 10-unit block would look at different distances. (The 5 -cm-wide standard block was shown at the four successive distances.)

Phenomenal size. You should try to estimate how wide the block would appear to be if it were along such a road or sidewalk. How wide a block would appear to be along a road may or may not be the same as how it actually would be if I were to measure it with a ruler. I'm not concerned with what the actual physical length of the blocks in the road would be, but rather with how wide they appear to be. Thus, your judgments should be based on the immediate appearance of size, taking distance into account, without concern with what might be the objective or actual size of the blocks on the receding surface. (These correspond most closely to what Carlson, 1977, calls "neutral apparent" instructions.)

Projective size. You should try to estimate how wide the block would be in terms of the width of the image it would project on your retina. Thus, as a block is moved further away it will project a smaller and smaller image on your retina. (The 5-cm-wide standard block was shown at the four successive distances, and it was pointed out that the image of this block gets smaller with increasing distance.) I am not concerned with how wide the block actually is, but rather how wide it would be projected on your retina.

Call the width of the sample block on the screen "10 units" wide. If you see a block of the same "retinal width," regardless of its distance or actual size, then it should also be judged to be 10 units wide. If you see a block that is wider than the 10 unit block, that is, wider in terms of the retinal image, then it should be given a larger number. Similarly, a block that projects a smaller image should be given a smaller number. Make your judgments proportional to the width of the retinal image projected by the block.

When three-space is projected onto two-space, retinal size corresponds to the "distal" size of the two-dimensional projection, and in judging retinal size, an observer can ignore contextual depth cues and respond directly to projected size. On the other hand, if the observer views the projection as a three-dimensional scene, the surface of the projection screen would be interpreted as a plane receding into depth; under such circumstances, retinal size is specified only as it is normally in three-dimensional scenes, and has no corresponding distal property. 
To examine the implications of this distinction we used two different instructional conditions to access proximal size. Projective instructions required observers to respond to the array as if it were a three-dimensional projection, and hence to take distance into account. To the extent that these instructions were followed, projective size was functionally equivalent to projective size in three-dimensional settings. Retinal-size instructions, on the other hand, required observers to ignore the context in which the stimuli were presented and to respond only to the size of the blocks on the screen. To the extent that observers can ignore contextual distance information, this latter condition should produce the closest approximation to retinal matching (i.e., the law of visual angle). The specific instructions were:

Retinal size. I would like you to judge the width of the block that will appear on each slide. Because of the receding surface, these blocks might appear to be at different distances on various trials. I want you to ignore how far away the block may appear to be. You should simply estimate how wide the block is on the screen. Completely ignore the receding surface. In other words, judge the width of each block as it appears in two dimensions, as if you were actually trying to measure, with a ruler, the width of the image on the surface of the two-dimensional screen.

After the experimental session, the subjects were asked to report their strategies for performing the task. Eight of the subjects were replaced because they verbalized judgmental modes other than the one instructed.

\section{Design}

The experimental design consisted of four instructional conditions (objective, phenomenal, projective, and retinal), four distances $(1.5,3.0,4.5$, and $6.0 \mathrm{~m})$, six distal block widths $(5,10,20,30,40$, and $50 \mathrm{~cm}$ ), two fixation points (top and bottom), and five replications. Instructions and fixation were between-group factors.

\section{RESULTS AND DISCUSSION}

Both the size judgment and chronometric data were subjected to overall analyses of variance. In addition, separate analyses were performed for each instructional condition. Due to the large number and power of the tests, a .01 level of significance was adopted.

\section{Size Judgments}

Figure 2 presents the mean judgments of size for each instructional set as a function of the ratio of the distal size of the comparison stimuli to that of the standard. For all conditions, size judgments increased systematically with distal size. The main effect of distal size ratio was statistically significant $[F(5,200)$ $=360.70]$, and separate analyses of variance indicated that this effect obtained for all instructions $[F(5,50) \geqslant 70.67]$. The overall main effect of instructional set was also significant $[F(3,40)=$ 12.82]. An orthogonal breakdown of this effect indicated that the combined size judgments for objective and phenomenal (distal mode) instructions $(\bar{X}=40.5)$ were reliably greater than the combined size judgments for the projective and retinal (prox-

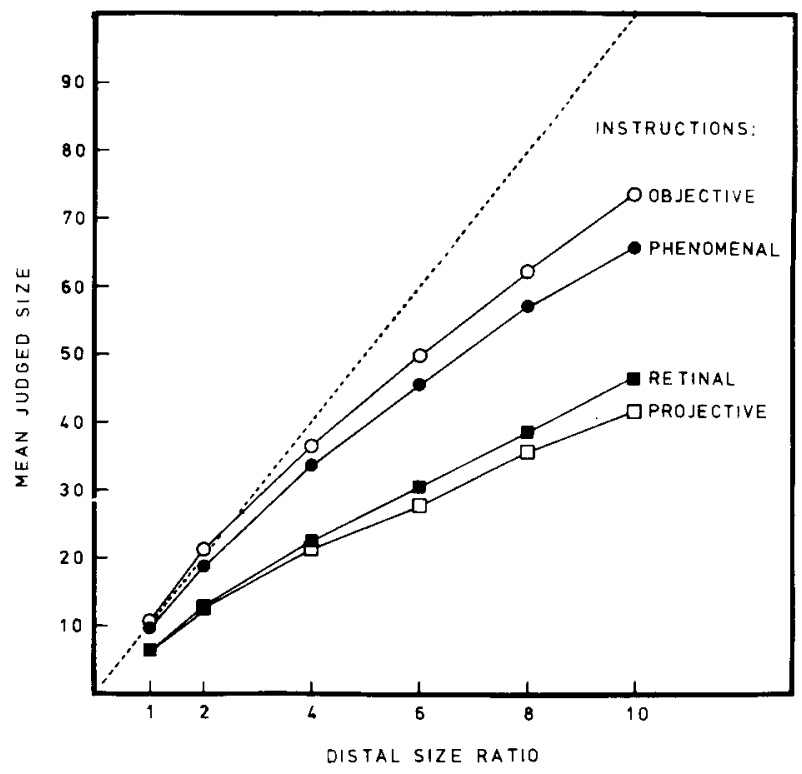

Figure 2. Mean judged size as a function of distal size ratio (i.e., the ratio of the size of the comparison stimulus to that of the standard) for the four instructional sets. The dashed line represents veridical distal matching.

imal mode) instructions $(\overline{\mathrm{X}}=25.2) \quad[\mathrm{F}(1,40)=$ 37.10]. The remaining orthogonal comparisons indicated that objective $(\overline{\mathrm{X}}=42.3)$ was not reliably different from phenomenal $(\overline{\mathrm{X}}=38.7)$ instructions and projective $(\bar{X}=24.1)$ was not different from retinal $(\bar{X}=26.2)$ instructions.

Distal size interacted with instructions $[\mathrm{F}(15,200)$ $=6.70]$, which is reflected as differences of the slopes of the four psychophysical functions shown in Figure 2. In addition, distal size interacted with distances $[F(15,600)=16.87]$. However, this did not hold for all instructional conditions. The Size by Distance interaction is shown for each instructional set in Figures 3a-3d. Superimposed size by distance functions, as is the case for phenomenal instructions in Figure 3b, indicates size constancy; i.e., perceived size is independent of distance. Divergence, or bilinear fanning, of these functions represents deviation from size constancy. For example, the divergence in Figures $3 \mathrm{c}$ and $3 \mathrm{~d}$ means that judged size decreased with distance (underconstancy) for projective and retinal instructions. Conversely, although the bilinear fan in Figure $3 a$ is only slightly divergent, the ordering is clearly inverted, indicating a tendency for judged size to increase with distance (overconstancy) for objective instructions. These different patterns in the size by distance functions across the four instructional sets produced a significant three-way Instructions by Size by Distance interaction $[F(45,600)=5.22]$. Separate analyses for each instructional set revealed that the Size by Distance interaction was significant for the projective 


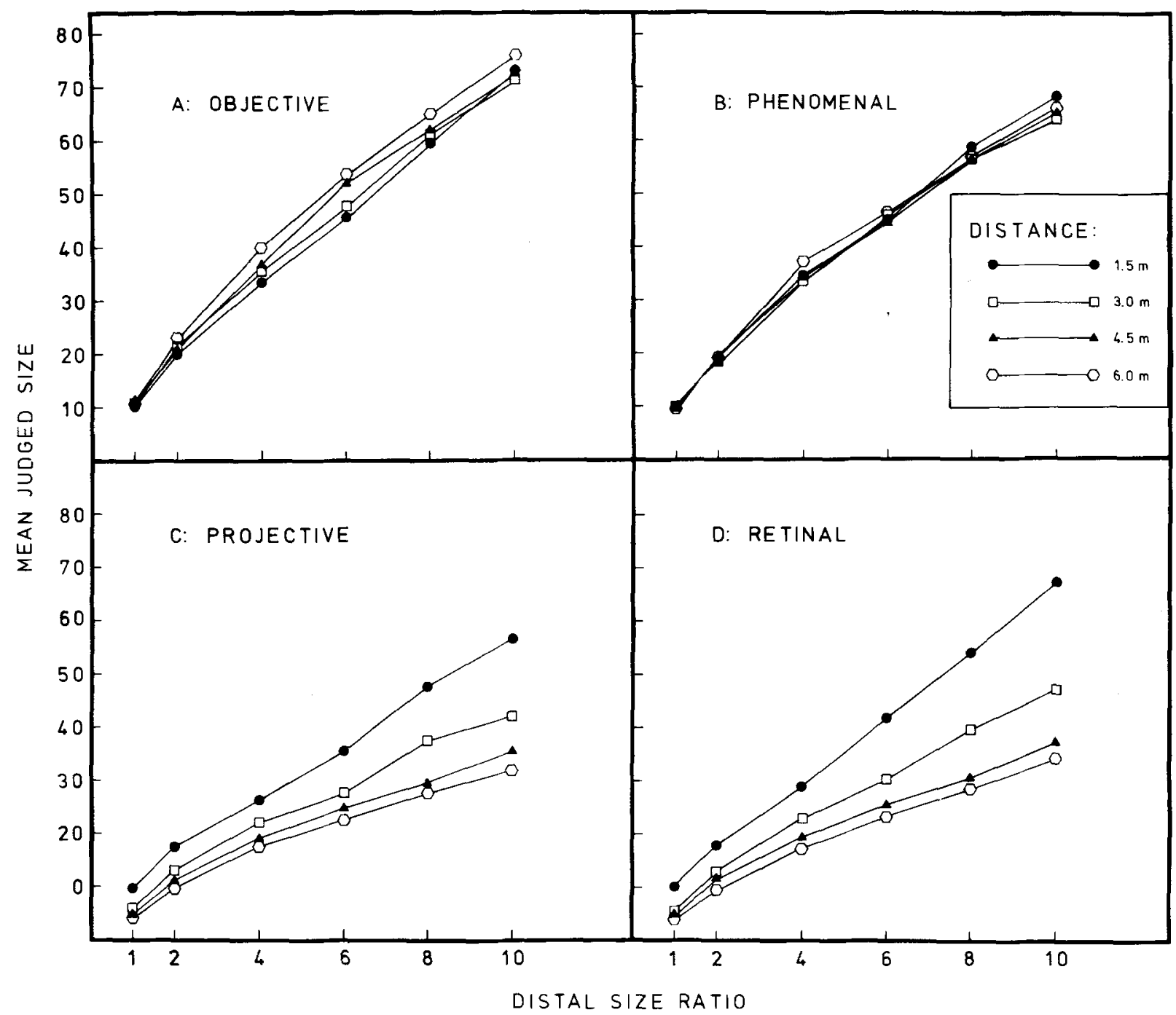

Figure 3. Mean judged size as a function of distal size ratio for each of the four instructional conditions (A, objective; B, phenomenal; $\mathbf{C}$, projective; $\mathbf{D}$, retinal) with distance as the curve parameter.

and retinal instructional sets $[F(15,150) \geqslant 5.57]$, but not for the objective or phenomenal instructional sets.

Overall judged size decreased with distance. However, the effect of distance also interacted with instructions $[F(9,120)=20.41]$, because although judged size decreased substantially with distance for projective and retinal instructions, it increased for objective instructions and did not vary systematically with phenomenal instructions. Individual analyses revealed significant main effects of distance for the objective, projective, and retinal instructions $[F(3,30) \geqslant 10.70]$, but not for the phenomenal instructions. The effects of distance for each instructional set are shown graphically in Figure 4. Each function in this figure is plotted in terms of the percent deviation of the mean size judgments for each distance from the mean size judgments at the closest distance to the observer (i.e., $1.5 \mathrm{~m}$ ). A failure to obtain size constancy occurs when judgments of size are affected by distance rather than when size estimation is simply nonveridical. Thus Figure 4 illustrates relative changes in size judgments as a function of distance. The dotted horizontal line indicates constancy, positive deviations from the horizontal line indicate overconstancy, and negative deviations, underconstancy. The dashed line in this figure represents retinal matching according to the law of visual angle. Figure 4 shows that there was overconstancy for the objective instructional set, close to perfect constancy for the phenomenal instructional set, and underconstancy for the projective and retinal instructional sets. (The interpretation of overconstancy for the 


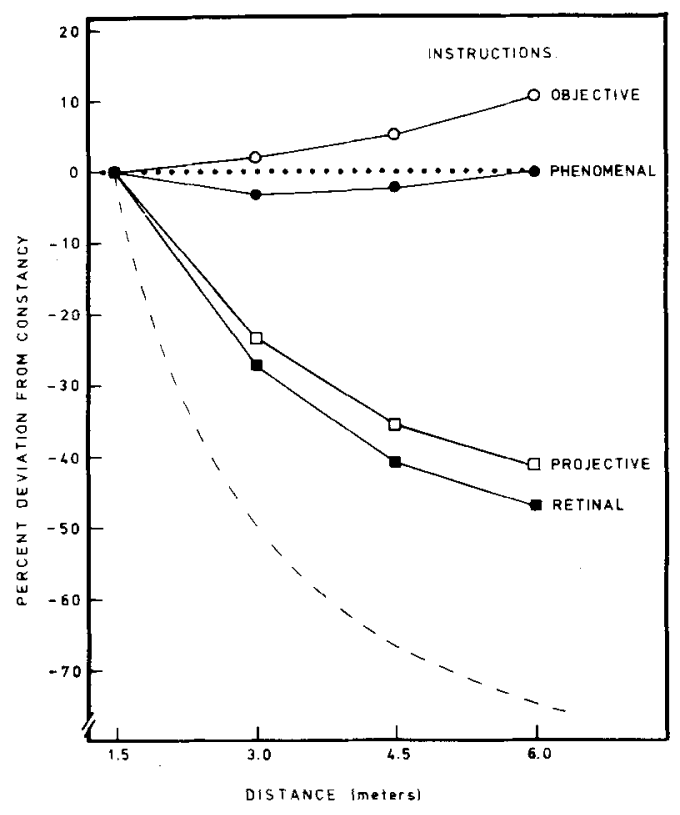

Figure 4. Percent deviation from relative constancy as a function of distance for each of the four instructional conditions. Relative constancy was defined as a percentage change from the pooled size judgments for the closest $(1.5 \mathrm{~m})$ distance. Thus, perfect constancy is represented by the dotted horizontal line indicating no change in size judgments as a function of distance, and perfect retinal matching, as defined by the law of the visual angle, is represented by the dashed negative exponential curve.

objective set must be tempered by the statistically nonsignificant bilinear fanning of the Size by Distance interaction shown in Figure 3a.)

The overall main effects of fixation $[F(1,40)<$ 1.00] and replications $[F(4,160)=2.29]$ were not statistically significant for judged size.

The general pattern of the judgmental results shown in Figures 2, 3, and 4 is very similar to a number of studies of size constancy involving threedimensional arrays (e.g., Brunswik, 1944; Carlson, 1962, 1977; Epstein, 1963; Gilinsky, 1955). The typical finding of these previous studies was that instructions systematically affected the degree to which perceived size remains constant with variations in distance. Specifically, phenomenal or "first look" instructions led to near-perfect constancy; objective instructions led to slight overconstancy; and projective instructions led to underconstancy that fell short of reintal matching. Replicating these findings in the present context suggests that the same size scaling processes evoked by real three-dimensional scenes also underlie perception of two-dimensional arrays, and supports Gibson's (1971) argument that the same kind of information that is found in the ordinary static environment is also inherent in pictures.

\section{Reaction Time}

There were three principal findings concerning the chronometric data. First, there were no systematic effects of distance on reaction time to make size judgments regardless of whether the observers were scaling proximal or distal size. These reaction time functions are shown in Figure 5. Neither the overall main effect of distance $[F(3,120)<1.0]$ nor the overall Instructions by Distance interaction $[F(9,120)$ $<1.0]$ was significant. The lack of a main effect for distance indicates that the processing of distance, per se, did not affect reaction time differentially.

Second, overall reaction times increased monotonically with the distal size ratio of comparison to standard $[F(5,200)=33.32]$. Figure 6 shows this increase for each instructional condition. Furthermore, the effect of size obtained for all instructional conditions $[F(5,50) \geqslant 3.64]$. Although inspection of Figure 6 suggests systematic reaction time differences due to instructions, this main effect did not reach statistical significance.

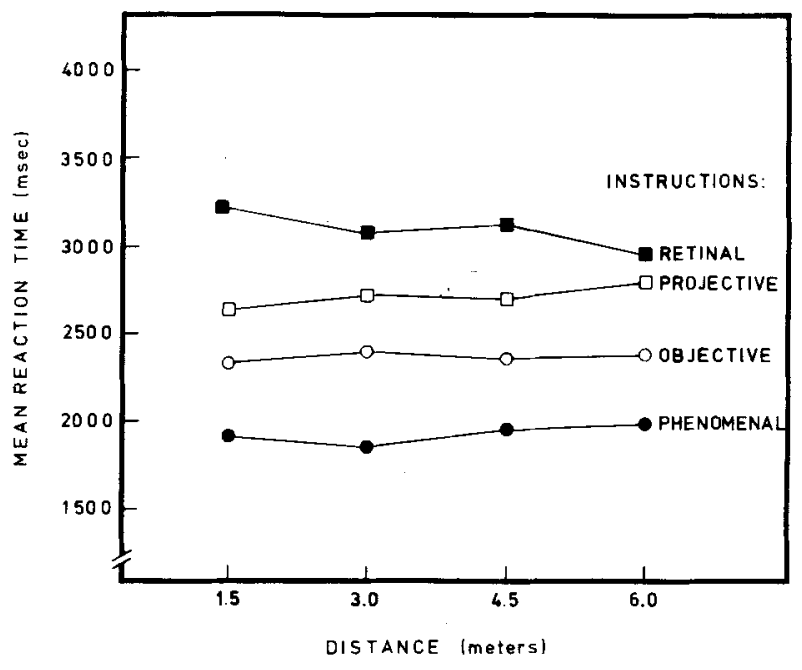

Figure 5. Mean reaction time as a function of distance for the four instructional conditions.

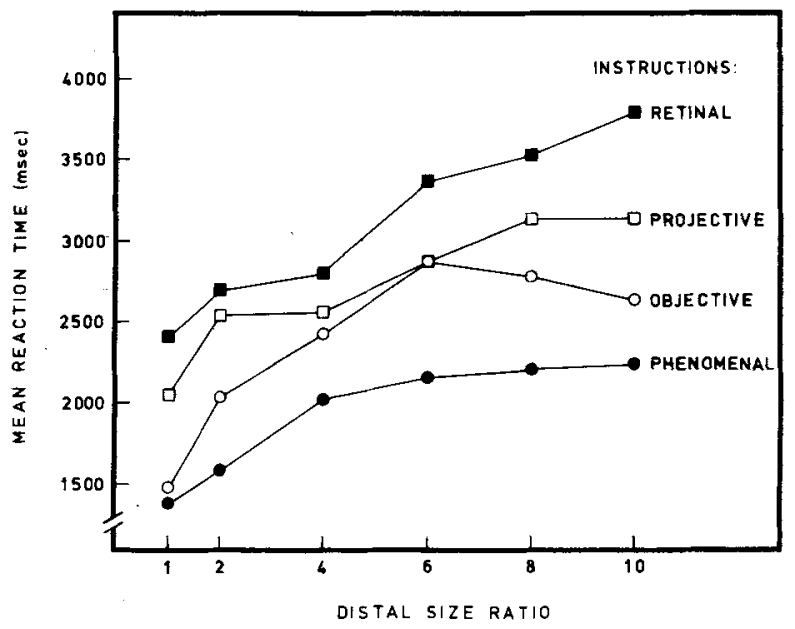

Figure 6. Mean reaction time as a function of distal size ratio for the four instructional conditions. 
An appealing interpretation of this second finding is that, in order to scale the size of an object relative to a standard unit, an observer must estimate the number of perceptual units in the comparison figure. The more perceptual units the comparison contains, the longer the time required. Nearly all subjects who were able to verbalize a strategy said they counted or estimated the number of standard figures that could be subtended by the test figure, a finding consistent with the notion that the same underlying transformational scaling process was used in all of the instructional conditions. These introspections are similar to those reported by Hartley (1977) in which the majority of subjects spontaneously reported that they formed an image of the standard length and laid it off along the line to be judged.

However, a third chronometric result suggested that there were some important differences in the type of scale or perceptual unit that was utilized in the various instructional conditions. Overall, the effect of size interacted with distance $[F(15,600)=$ 4.07]. However, a significant three-way interaction $[F(45,600)=2.03]$ indicated that the nature of the two-way interactions depended on instructions. Figures 7a-7d illustrate the Size by Distance interactions for each instructional condition. Individual analyses revealed that the Size by Distance interaction was significant for projective (Figure 7c) and retinal (Figure $7 \mathrm{~d}$ ) instructions $[\mathrm{F}(15,150) \geqslant 2.37]$, but nor for objective (Figure 7a) or phenomenal instructions (Figure 7b). This finding suggests that instructions altered the nature of the perceptual scale unit. Distal mode instructions required scaling the distal sizes of the comparison relative to a scale unit of a constant distal size. A figure of fixed distal size subtended the same number of scale units at all distances, even though its proximal size diminished as distance increased. This is consistent with the functions in Figures $7 \mathrm{a}$ and $7 \mathrm{~b}$, which show that

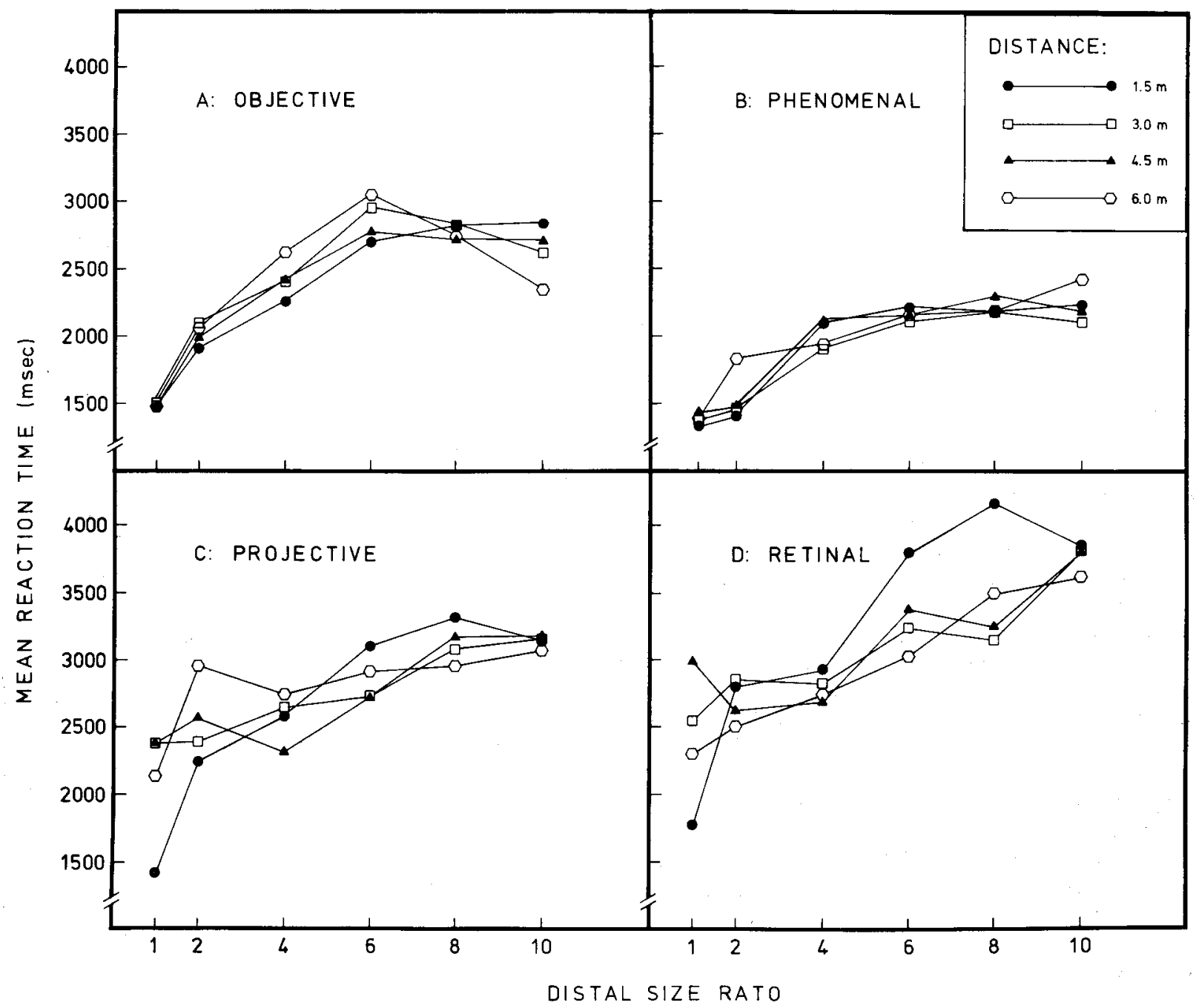

Figure 7. Mean reaction time as a function of distal size ratio for each of the four instructional conditions (A, objective; $B$, phenomenal; $C$, projective; $D$, retinal) with distance as the curve parameter. 
reaction time increased as a function of distal size ratio, and that variation in proximal size due to distance had no effects.

On the other hand, the proximal mode instructions required scaling of the proximal sizes of the comparison figures relative to a standard scale unit. In principle, scaling in these conditions is independent of distance in that observers had only to scale the two-dimensional width of the projected image, and therefore a scale unit would be of invariant proximal size at all distances. This would mean that any stimulus of fixed distal size should subtend fewer scale units with increasing distance, and as a consequence the times associated with the scaling process should decrease with distance if counting or estimating perceptual units is involved. Such an effect would be manifested as changes in the slopes of the reaction time functions in Figures $7 \mathrm{c}$ and $7 \mathrm{~d}$. If perceived size followed the law of the visual angle in these conditions, the slopes should decrease with distance according to the progression $\mathrm{x}, .5 \mathrm{x}, .33 \mathrm{x}$, and $.25 \mathrm{x}$ for the distances from 1.5 to $6 \mathrm{~m}$. Judgments in these conditions did not attain the law of the visual angle, and while the slopes of the reaction time functions tended to decrease with distance in Figures $7 \mathrm{c}$ and $7 \mathrm{~d}$, the decreases were localized mostly between the $1.5-$ and $3-\mathrm{m}$ distances. For example, the slopes associated with the four distances in Figure $7 \mathrm{c}$ are 184, 94, 102, and $68 \mathrm{~m} / \mathrm{sec}$, respectively. ${ }^{1}$ In contrast, the slopes of the reaction time functions were equivalent for all four distances in the distal mode conditions (Figures 7a and $7 \mathrm{~b}$ ), presumably because each distal size always contained the same number of scale units.

In order to estimate size, it appears that the observer must scale the linear extent of the comparison in relation to the perceptual scale unit. Instructions determined if the scale unit was of constant distal size (objective and phenomenal instructions) or of constant proximal size (projective and retinal instructions) as a function of perceived distance in the two-dimensional array. The idea that observers count or estimate the number of scale units in a comparison figure is similar to Hartley's (1977) notion that observers judge line length by laying off a mental image of the standard against the comparison. Both studies found a monotonic increasing relation between reaction time and size.

The overall increase in reaction time as the ratio of test stimulus to standard stimulus size increased is similar to results obtained in a number of studies investigating size-invariant shape recognition (Besner \& Coltheart, 1976; Bundesen \& Larsen, 1975; Sekuler $\&$ Nash, 1972). In these studies, the observers made same-different judgments of shapes, and in order to do so had to transform, or normalize, the "irrelevant" dimension of the size of the test figures.
In all cases, the stimuli were two-dimensional with no pictorial cues for depth, and therefore the proximal and distal sizes of any given stimulus were equivalent. These studies showed linear increases in reaction times as a function of the size ratio of the standard and comparison figures similar to the present study. An important methodological difference, however, was that in the previous studies the observers implicitly scaled (normalized) size in order to make judgments regarding shape, whereas in the present study the observers were explicitly required to make size scaling judgments. Nevertheless, the overall similarity of the reaction time functions suggests that similar transformational processes may underlie both kinds of scaling.

The overall main effects of fixation and replications were not statistically significant; however, fixation interacted with distance $[F(3,120)=5.43]$. Individual analyses revealed that this interaction was significant only for retinal instructions $[F(3,30)=$ 6.13]. This interaction may reflect the effects of systematic eye movements. The purpose of the retinal instructional set was to have the observer judge the widths of blocks on the two-dimensional plane of the viewing screen and ignore the context of the receding array. Thus, for retinal instructions, "distance" in the Fixation by Distance interaction is actually the vertical distance on the two-dimensional plane of the viewing screen and not perceived distance on a receding array. A crossover interaction involving fixation point and distance should obtain if the time to scan the array vertically was important. Inspection of the mean time associated with the different distances for the two fixation conditions of retinal instructions bears this out. For the top fixation condition, the means were $3,489,3,984,3,918$, and 4,371 msec for the "distances" from closest to the furthest from the top fixation point. Similarly, the means were $2,070,2,231,2,273$, and $2,415 \mathrm{msec}$ for the "distances" from the closest to the furthest from the bottom fixation point. In other words, in retinal instructions, the fastest mean reaction time to make size judgments was for the distance (on the screen) closest to the fixation point.

In the overall analyses, the remaining interactions were not statistically significant.

Epstein and Broota (1975; cf. Broota \& Epstein, 1973) examined the reaction time associated with size scaling in three-dimensional arrays and obtained results generally inconsistent with our findings. Epstein and Broota reported no effects of size on response times. Instead, response times increased as a function of the distance from the observer to the target stimulus for objective instructions, but not for phenomenal instructions. Epstein and Broota suggested that the effect of distance was due to additional cognitive processing associated with overconstancy, 
and since phenomenal instructions did not lead to overconstancy, the corresponding reaction time function did not increase with distance.

The lack of an effect of stimulus size on response time is, of course, inconsistent with both Hartley (1977) and the present study, and it is possible that the effects of distance obtained by Epstein and Broota may have been due to extraneous factors. For example, they did not attempt to control for the times required for accommodation and vergence eye movements made in fixating objects at different distances; it may have been that these peripheral adjustments affected reaction times for the objective instructional condition, but that the phenomenal instructions encouraged the observers not to wait for such adjustments. The remarkably fast times (in the range of $240 \mathrm{msec}$ ) reported for Epstein and Broota's phenomenal instructional condition supports this argument and suggests that their phenomenal instructions might have overemphasized speed. The present study eliminated possible confounding influences of accommodation and vergence eye movements by varying perceived distance via pictorial depth cues so that all stimuli were presented in a single depth plane. Also, in their experiment, the subjects pressed the key that stopped the clock simultaneously with making a verbal size estimate. Such a procedure has been criticized on the grounds that subjects are able to press the key prior to the termination of mental processing (cf. Woodworth, 1938). The use of an electronic voice key in conjunction with the requirement of smoothly articulated verbal size estimates guarded against this possibility in the present experiment.

\section{GENERAL DISCUSSION}

Gibson's $(1950,1966)$ approach to space perception provides a framework than can encompass the relations we obtained between instructions, size constancy, and reaction time. According to Gibson, size constancy "is a by-product of the constant scale of the visual world at different distances. Scale, not size, is actually what remains constant in perception" $(1950$, p. 181 , italics ours). In other words, it is Gibson's position that a gradient of texture and other size perspective information provide a constant scale against which sizes are evaluated. A constant distal size subtends a constant number of textural units; when linear perspective is well defined, the horizontal extent of the stimulus subtends a constant proportion of the array irrespective of position in the array. Size is judged in relation to some unit, and in a perspective array this relation is invariant. Hence, distal size is immediately accessible to the observer. In this regard, it is interesting to note that the absence of an effect of distance on reaction time is consistent with Gibson's (1950) notion that scale is present at all perceived distances in the optical array. Of course, if distal size can be scaled directly, it would not be necessary for observers in the distal mode instructional conditions to first scale proximal size and then somehow transform that value in terms of perceived distance.

The effects of instructional sets in the present study would be to induce the observers to define different types of scale units against which size is measured. Within such a framework, distal mode instructions induce a scale unit of constant distal extent, that is, a unit that is preserved in the size perspective information inherent in the array. This contextual information provides the constant scale against which all distal size estimates are to be made. On the other hand, the proximal mode instructions induce a scale unit of constant proximal size, and in this case an invariant scale is not explicitly available throughout the array. In fact, contextual information is in direct opposition to a scale unit of constant proximal size. Assuming that observers are able to construct an invariant scale which has a unit of constant proximal size (e.g., some constant proportion of the entire visual field), the size of a test figure would be measured or scaled relative to this perceptual unit. Thus, we are suggesting that instructions affect the nature of the scale unit against which the measurement transformations are performed, but do not directly affect the size scaling or measuring process itself.

Even though the differences are not statistically significant, it is interesting to note that reaction times generally increased as deviation of size judgments from constancy increased. The overall mean percent deviations from constancy were $.8,5.9,33.5$, and 38.3 for phenomenal, objective, projective, and retinal instructions, respectively, and the corresponding mean reaction times were $1,926,2,367$, 2,716 , and $3,094 \mathrm{msec}$. Thus, phenomenal instructions produced size constancy and the fastest overall reaction times. Retinal instructions, on the other hand, produced the greatest deviation from constancy as well as the longest overall reaction times. If one assumes that a distal scale unit is directly specified at all points in the array and that size judgments are made relative to such a scale unit, then distal size would be relatively straightforward and hence more quickly attained. Size judgments based on a scale unit other than one that is specified in the array would require constructing the requisite scale as well as ignoring the scale that is already provided. Such operations would no doubt require additional cognitive processing and therefore be associated with longer reaction times. This is similar to Carlson's notion (e.g., 1977) that distal 
size is available prior to projective or objective size and that both projective and objective judgments require additional operations. Carlson, however, apparently believes these operations to be judgmental biases away from the more immediate perception of distal size, whereas our position is that these operations are based on the same type of processes that allow one to attain distal size.

In summary, the present paper examined size constancy evoked by a two-dimensional pictorial array. Size judgments and response times were consistent with a general scaling strategy that was independent of both the instuctional condition and the distance of the comparison stimulus. The effect of instruction was to influence the nature of the scale unit adopted by the observer. The observer's utilization of the scale unit as a basis of perception, in conjunction with the lack of effects for distance on response time, is compatible with Gibson's notions of size perception.

Accordingly, the sizes of objects at a given distance are perceived relative to the scale unit as it is defined for that distance. Under normal circumstances, constancy will prevail, since environmental cues provide a type of scale that is constant as a function of distance. However, the observer is probably capable of using a variety of different scales to measure (transform) size with variation of distance. Any such scale must operate against the pervasive contextual information that is provided under conditions of normal viewing. Virtually all contextual information in the present experiment favored a unit of constant distal size. Not only did the texture elements of the array favor such a unit, but certain features of the comparison stimuli were also consistent with an invariant distal unit. For example, each stimulus block projected into depth, and the height and depth of the blocks varied according to linear perspective and foreshortening, respectively. In the proximal instructional conditions, the observer was asked to ignore the depth cues associated with each comparison stimulus and to judge only horizontal extent. There is ample evidence that observers are unable to completely ignore certain irrelevant dimensions (e.g., Dixon \& Just, 1978; Garner, 1974, 1976). Figure 3 shows that proximal mode instructions led to size judgments that fell short of the law of visual angle, suggesting that constructing a scale unit that is completely independent of irrelevant perspective cues may be impossible.

\section{REFERENCES}

Besner, D.. \& Coltheart, M. Mental size scaling examined. Memory \& Cognition, 1976, 4, 525-531.

Broota, K. D., \& Epstein, W. The time it takes to make veridical size and distance judgments. Perception \& Psychophysics, 1973, 14, 358-364.
Brunswik, E. Distal focusing of perception: Size constancy in a representative sample of situations. Psychological Monograph, 1944, 56, Whole No. 254.

Bundesen, C., \& Larsen, A. Visual transformation of size. Journal of Experimental Psychology: Human Perception and Performance, 1975, 1, 214-220.

Carlson, V. R. Size constancy judgments and perceptual compromise. Journal of Experimental Psychology, 1962, 63, 68-73.

Carlson, V. R. Instructions and perceptual constancy judgments. In W. Epstein (Ed.), Stability and constancy in visual perception. New York: Wiley, 1977.

Dixon, P., \& Just, M. A. Normalization of irrelevant dimensions in stimulus comparisons. Journai of Experimental Psychology: Human Perception and Performance, 1978, 4, 36-46.

Epstein, W. Attitude of judgment and the size-distance invariance hypothesis. Journal of Experimental Psychology, 1963. 66, 78-83.

Epstein, W., \& Broota, K. D. Attitude of judgment and reaction time in estimation of size at a distance. Perception \& Psychophysics, 1975, 18, 201-204.

GARNER, W. R. The processing of information and structure. Potomac, Md: Erlbaum, 1974.

GaRNER, W. R. Interaction of stimulus dimensions in concept and choice processes. Cognitive Psychology, 1976, 8, 98-123.

Ginson, J. J. The perception of the visual world. Boston: Houghton Mifflin, 1950.

G1Bson, J. J. The senses considered as perceptual systems. New York: Houghton Mifflin, 1966.

Gibson, J. J. The information available in pictures. Leonardo, $1971,4,27-35$.

GiLinsky, A. The effect of attitude on the perception of size. American Journal of Psychology, 1955, 68, 173-192.

HAGEN, M. A. An outline of an investigation into the special character of pictures. In H. L. Pick \& J. E. Saltzman (Eds.), Modes of perceiving and processing information. Hillsdale, N.J: Erlbaum, 1978.

Hagen, M. A., \& Jones, R. K. Cultural effects on pictorial perception: How many words is one picture really worth? In R. D. Walk \& H. L. Pick (Eds.), Perception and experience (Vol. VI). New York: Plenum, 1978.

HaRTLEY, A. A. Mental measurement in the magnitude estimation of length. Journal of Experimental Psychology: Human Perception and Performance, 1977, 3, 622-628.

Se kuler, R., \& NASh, D. Speed of size scaling in human vision. Psychonomic Science, 1972, 27, 93-94.

Smith, O. W., \& Gruber, H. Perception of depth in photographs. Perceptual \& Motor Skills, 1958, 8, 307-313.

Smith, O. W., Smith, T. C., \& Hubbard, D. Perceived distance as a function of the method of presenting perspective. American Journal of Psychology, 1958, 71, 662-675.

Teghtsoonian, R., \& Teghtsoonian, M. The effect of size and distance on magnitude estimations of size. American Journal of Psychology, 1970, 83, 601-612.

WoodworTh, R. S. Experimental psychology, New York: Holt, Rinehart, \& Winston, 1938.

\section{NOTE}

1. The fastest reaction times within a given instructional condition should occur when the comparison figure is one "scale unit" wide. If the comparison figure subtends more or less than one scale unit, its judged size must be based on some transformational or scaling process relative to the unit. In distal mode instructions, the size of the smallest comparison $(5 \mathrm{~cm})$ was always distally equivalent to the size of the standard unit and, accordingly, was associated with the fastest reaction times (Figures $7 \mathrm{a}$ and $7 \mathrm{~b}$ ). In the proximal mode instructions, however, the distal stimulus size that was equivalent to the proximal scale unit changed with distance. Specifically, the $5-\mathrm{cm}$ standard was 
proximally equivalent to the $5-, 10-, 15-$, and $20-\mathrm{cm}$ comparisons at the viewing distances of $1.53 .0,4.5$, and $6.0 \mathrm{~m}$, respectively. Thus, each of these comparisons subtended one proximal scale unit. This relation should be reflected in Figures $7 \mathrm{c}$ and $7 \mathrm{~d}$ as a series of "V-shaped" functions, with the vertex of each function occurring at the distal size perceived to subtend a single proximal scale unit. As distance increases, the vertex should shift to the larger distal size values referred to above. Such functions are not apparent in Figures $7 \mathrm{c}$ and $7 \mathrm{~d}$. However, few subjects gave proximal size judgments that followed the law of visual angle (see Figure 4). Consequently, distal sizes other than 5. 10, 15, and $20 \mathrm{~cm}$ (at the four respective distances) could have been perceived to subtend a single proximal scale unit. The vertex would depend on what values were perceived equivalent to the proximal unit. If these values differed for different observers, then the overall lack of vertices is not surprising. Examination of the data for individual subjects revealed a high proportion of such $\mathrm{V}$ functions in retinal and projective instructional conditions $(51.4 \%)$, a lower proportion for phenomenal instructions $(27.8 \%)$, and a still lower proportion for objective instructions $(2.8 \%)$.

(Received for publication March 15, 1979; revision accepted October $17,1979$. 